LSP International Journal, Vol. 8, Issue 1, 2021, 19-34

(C) Universiti Teknologi Malaysia

E-ISSN 2601-002X

DOI: https://doi.org/10.11113/lspi.v8.16787

\title{
Material Design for Teaching Citations Skills in Scientific Writing
}

\author{
Kobra Mansourizadeh \\ Faculty of Humanities, Apadana Institute of Higher Education, Shiraz, IRAN \\ Ummul K. Ahmad \\ Language Academy, Faculty of Social Sciences and Humanities, Universiti Teknologi Malaysia, \\ 81310 UTM Johor Bahru, Johor, MALAYSIA
}

Submitted: 30/3/2021. Revised edition: 5/5/2021 Accepted: 24/5/2021. Published online: 28/6/2021

\begin{abstract}
Citations are important rhetorical devices that when properly and strategically employed, allow writers to promote their current research findings persuasively and efficiently. As knowledge construction is progressive and cumulative, specifically in scientific disciplines, it is evident that acquiring skills for adequate and effective application of citations is essential for success. Scientific writers are required to possess advanced academic literacy skills in order to ably position their study within the framework of existing knowledge, and strategically employ citations to advance the acceptability of their research findings. This paper endeavors to propose materials for teaching the rhetorical functions of citations in advanced academic writing courses. The tasks are designed specifically to raise students' awareness of citation norms especially in their own specific disciplines. Since the practice of citing work of others ethically is highly challenging for emerging research writers, materials suggested in this paper can be beneficial to instructors who are involved in developing advanced discipline-specific writing courses, or short-term academic writing workshops.
\end{abstract}

Keywords: Advanced Academic Writing, Citations, Material Design, English for Research Purposes

\subsection{INTRODUCTION}

Advanced academic writing is a literacy practice that is shaped by the disciplinary conventions of specific communities. Academic tasks at post-graduate levels demand advanced literacy skills beyond the basic academic reading and writing in the discipline. Research writing demands not only disciplinary content knowledge but also sophisticated knowledge on rhetorical features of academic writing. As advanced writing practices are shaped by disciplinary conventions, it thus requires knowledge on established and appropriate strategies specific to the discipline and ways in which to present the writing convincingly and persuasively. Indeed, rhetorical and written interactive features have long been emphasized in scholarly and scientific writing literature (see Myers, 1990; Swales, 1990;

*Correspondence to: Ummul K. Ahmad (email: m-ummul@utm.my) 
2004; Chau, 2017; Li \& Zhang, 2021). Scientific writing then is not simply manifestation of facts but also a form of social interaction within the disciplinary communities (see Latour and Woolgar, 1979; Bazerman, 1988), making the roles of rhetorical features become important. In other words, scientific writers need to have proper rhetorical knowledge to show the significance and value of their research and make their writing more persuasive to their target readers. Academic writers need to strategically advance the acceptability of their research claims as "persuasion is at the heart of science" (Bazerman, 1988, p.88) and citation is "central to the social context of persuasion" (Hyland, 1999, p. 342). Citation thus provides various rhetorical means for research writers.

As has been established in the literature, scientific communities are based on the creation of new knowledge or new facts (Bazerman et al., 2005). Researchers use prior knowledge provided by previous research and build new knowledge based on the old ones. Scientists, based on their research findings, attempt to offer new claims in relation to pre-confirmed and accepted facts already established in the disciplinary community. For their claim to be accepted as new knowledge and become a fact, it needs to be approved by the members of the community. To gain acceptance, research writers need to contextualize their findings in relation to previous works of others within their specific field of study and present their findings by following specific conventions of the community. Claims made in scientific writing need to be convincing and adequately supported to gain community approval (Hyland, 1996). To achieve this purpose, citations are usually employed as they serve as 'evidence' that enhance the acceptability of the claims (Bazerman, 1988).

One of the specific features of scientific research is "cooperative endeavor" (Bazerman, 1988, p.303)—researchers in communities of the same interest endeavour to solve problems by building on previous knowledge. Thus, knowledge becomes cumulative and incremental in the field; in other words, knowledge advancement has a linear growth (Hyland, 1998) and especially so in the hard disciplines. Therefore, citation or 'manifest intertextuality' (Hyland, 1999, 2000) plays a crucial role in attracting interests of the community. By showing the relations with what has been established earlier and by emphasizing the novelty of the current research findings, research writers persuade their target readers towards accepting new claims being made.

For emerging writers, however, the writing strategies and rhetorical practices may not be so apparent; young researchers who are considered apprentices in the field may yet to learn how to navigate their readings of disciplinary texts or learn how texts are constructed and negotiated within the field. Novice writers often require direct access to expert members in their discourse community who would be willing to mentor them with disciplinary practices (Wenger, 1998). This access could be provided in the form of sharing and creating various artifacts that are specific to that social group. For instance, in a community of engineering researchers, the dominant artifact of major interest and paramount importance is the research paper. The established academic writing conventions of a specific social group or community of practice are usually deeply embedded in research papers produced by senior, established members of the community. Nonetheless, some of these academic practices which form the culture and identity of the community are not instantly visible nor easily discernible to novices or beginning research writers. Since visibility plays an important role in successful apprenticeship (Lave and Wenger, 1991), it is crucial that novice writers whose academic skills are still developing be consciously directed to learn the necessary skills. 
Novice research writers in any scientific community could be helped to understand the appreciated academic writing practices by making the practices clearly perceptible for them. Many rhetorical features of academic texts, including the intricacies of citations, for instance, are not visible at the surface level. Citation is often described as an 'occluded feature of academic writing' (Pecorari, 2003, 2006). Hence further scrutiny beyond the surface level is usually needed to reveal the citation practices that have been structurally applied and organized by expert writers in their published papers, before those practices can become visible and easily learned and adopted by novice writers of the same community. However, novice writers might not be able to make full use of citation as a rhetorical device to make their text more persuasive if they have yet to be fully aware of the rhetorical conventions of the field. Flowerdew (1999) has also identified that indeed the abstract aspects of academic writing such as rhetorical features and reporting of the literature as the main problems commonly faced by beginning writers, particularly from non-English language background who are embarking on writing research with the purpose of publication. Therefore, there is a need to raise students' awareness on how to employ citations effectively and strategically in advanced academic writing instructions.

Due to the crucial role of citation in scientific writing, applied linguists have been analysing various aspects of how citation are employed by academic writers across different disciplines: for example, Hyland (1999) has focused on disciplinary differences of citation that are used in the humanities and social sciences as opposed to the hard disciplines; Charles (2006) has studied reporting clauses in citations in the fields of politics and material science. Thompson and Tribble (2001) have investigated citation types in doctoral theses in the fields of agricultural botany and agricultural economics while Thompson $(2000,2005)$ has reported on citation practices in PhD theses in the field of agricultural botany. Petrić (2007) focuses on citation functions in master's theses in gender studies while Zaid, Shamsudin, and Habil (2017) investigated the applications of citations in doctoral theses in the field of chemical engineering. Li and Zhang (2021) analyze and compare citation competence of Chinese master's and doctoral students' theses in a sub-field of English, specifically in the literature review and discussion segments. Harwood (2009) has reported the functions of citations used by sociologists and computer scientists while Hewings et al. (2010) have examined the geolinguistic aspects of citations practiced by scholars in the field of psychology. Mansourizadeh and Ahmad (2011, 2016) have compared the citation practices in novices versus expert engineering writers and Shooshtari et al. (2017) look into the citation features in mechanical and computer engineering and compare them with ones from applied linguistics and psychology.

All these studies have called for specific pedagogical applications for teaching emerging research writers on the intricacies of citing strategies and citations patterns in general. Various other studies have also shown the novice writers' challenges in appropriate application of citations in their research papers (see e.g., Pecorari, 2003, 2006; Abasi et al., 2006; Keck, 2006; Flowerdew and Li, 2007; Shi, 2010; Ahmad, Mansourizadeh and Koh, 2012). Academic writing instructors should focus on pedagogical methods which involve students in different textual practices as have been signified in the literature (see Pecorari, 2003; Carroll, 2004; Howard 2002, 2007; Abasi and Akbari, 2008; AdlerKessner et al. 2008; Robillard and Howard, 2008). We need to raise the students' awareness of details of citation norms, draw their attention to the appropriate use of citations, and familiarize them with the citing behaviour of scholars in their field of study and the genre of research articles. Clearly, 
'awareness' and 'exposure' have been identified as the two factors that could help students with their academic tasks (Johns and Swales, 2002).

In this paper, we attempt at designing materials and tasks which can help raise students' awareness of rhetorical functions of citations in research papers and to promote their skill of citation use. The materials and tasks can easily be adopted as a part of semester-long academic writing courses or short-term academic writing workshops. The instructional materials are designed following the EAP tradition (c.f. Swales and Feak, 2010) of raising students' awareness, involving them in actual text analysis, and enhancing their acquisition of the targeted subject. Through exercises presented in the following sections, students can be made aware of different rhetorical functions of citations, be actually involved in their own citation analysis, and be guided on how to review the citations in their own writing.

\subsection{RAISING AWARENESS OF RHETORICAL FUNCTIONS OF CITATIONS}

Novice students usually refer to published articles in order to familiarize themselves with the writing conventions of their discipline. However, as we have pointed out earlier, many citation practices are not evident from merely reading the published text. Therefore, it is significant to draw students' attention towards the rhetorical features at the very first stage of acquiring citation skills. During this stage, the instructor provides different examples of citations for the students to raise their awareness of the various rhetorical functions of citations. The instructor also provides some key linguistic words which can help them to identify citation functions. Some of the functions are linguistically and clearly identifiable, while others may require students to actually refer to the cited text, or even to the neighboring sentences. The students are also informed that sometimes a citation serves different purposes; hence it can be associated with different rhetorical functions. Several common functions of citations have been identified by different researchers including source, identification, reference, and origin (Thompson and Tribble, 2001; Thompson, 2005), attribution, exemplification, further reference, statement of use, evaluation, comparison of one's own findings with other sources, and establishing links between sources (Petrić, 2007). In this paper, we have consolidated all these functions into seven distinctive categories. For the sake of clarity, we provide a brief description and an example for each function. All the examples provided in the following sections are taken from actual research papers published in the field of chemical engineering where citations are used in form of numbers in brackets (see Appendix A for the list of journals from which the research papers are retrieved).

\subsection{Attribution}

Citations with the function of attribution are used to provide background knowledge of the field or to report previous research findings. The name of the cited author could be used as a part of the cited sentence or it could be referred to in parentheses or as a number in brackets. The citation could be: 
a) Reporting: In reporting citation, there is a reporting verb.

Stoyanoz reported that tensile strength dry (TSD) of acrylic fibers showed a maximum at a certain polymer concentration [9].

b) Non-reporting: In non-reporting citation, there is no reporting verb (the citation is used to provide background knowledge).

The mass transfer between the spun fibers and the surrounding medium influences microscopic and morphological structures as well as the mechanical properties of PAN fibers [6].

c) Adjunct 'according to': A citation could also be of attribution function when it begins with adjunct 'according to'.

According to Knudsen [10], an increase in the dope solid in the range of 15-25\% improved the homogeneity of the fiber structure by reducing the generation of large voids.

A citation is categorized as attribution only if no other functions could be identified for it.

\subsection{Identification}

Citations with identification function are used to identify the agent or the actor of the verb in the sentence (e.g. is reported, is argued, is discussed). The sentence is in passive mode and the cited author is referred to in parentheses/brackets.

The peak with BE of $288.7 \mathrm{eV}$, is attributed to $C-S$ species [24].

Citations with identification function are also used to identify various studies which have investigated different materials. These citations usually consist of the name of some specific materials.

Fluorinated polymer films such as polytetra-fluoroethylene (PTFE) [7-9], poly(tetrafluoroethyleneco-hexafluropropylene) (FEP) [10-19], poly(ethylene-alt-tetrafluoroethylene) (ETFE) [20,21] and poly(vinylidene fluoride) (PVDF) [22-25] have been extensively used as substrates to develop sulfonic acid membranes.

\subsection{Comparison}

Citations with the function of comparison are used to compare one's own results with that of the previous research to highlight the similarities or dissimilarities. This type of citation can be identified by the comparison language that is used (e.g. similar to, different from, on the contrary, in agreement, etc.). This citation function is only used in the results and discussion section where the findings of research are presented. 
The trend of the results in the present grafting system is similar to that reported for grafting of styrene diluted with benzene and containing 0,2 and 4\% DVB onto FEP films using simultaneous and preirradiation methods [18].

\subsection{Reference}

Citations with the function of reference are used to lead the reader to a source for further reading. They are used to save space in the paper (sometimes they are indicated by directive 'see'). This function of citation can be identified by the language that is used which directs the reader to additional sources for reading (e.g. discussed elsewhere, reported earlier, covered in other literature, etc.).

Different methods of polymer membrane preparation have been covered in several reviews [3]

Details of the effect of the various reaction parameters on the degree of grafting and crosslinking of styrene/PFA system are thoroughly discussed elsewhere [31].

\subsection{Establishing Links between Different Sources}

This citation function is used to establish a link between studies with similar focus, findings, or argument. This citation could easily be identified as it ascribes the information to more than one source:

a) Sources of similar focus:

Humic acids have been studied by a number of researchers as a model compound of NOM in water [35].

b) Sources of similar findings:

Sulfonic acid membranes prepared by radiation-induced grafting have been found to be potential substitutes for their corresponding commercial fuel cell membranes [2-4].

c) Sources of similar argument:

The formation of crystallite phases is due to the high metal loading on the support that has weakened the metal-support interaction [29, 39]. 


\subsection{Support}

This citation is used to provide support for the topic of the study, the methodology used, or to justify the findings and the claims made.

Membrane systems for natural gas treating offer significant advantages, such as flexibility in design, compactness, simplicity and efficiency which are superior over conventional gas separation methods [3].

The above citation provides support for the topic under investigation and shows the significance of the study.

The dope temperature was maintained at $22^{\circ} \mathrm{C}$ in order to reduce skin formation due to substantial temperature different during coagulation process [14].

The citation used in the example above provides support for the methodology and the procedures employed by the authors.

In sharp contrast, membrane annealed at $180^{\circ} \mathrm{C}$ shows an immediate increase of the pressurenormalized flux by a factor of 8 with increasing feed pressure. Again, this suggests that vigorous backbone mobility tends to destroy any induced molecular order at $180^{\circ} \mathrm{C}$ or above this heating temperature [24].

In the first sentence above, the writers explained parts of their findings and in the second sentence, they justified the findings by providing a citation.

\subsection{Multifunctional Citations}

Sometimes a citation is used with different functions hence referred to as multifunctional.

Fluorinated polymer films such as polytetra-fluoroethylene (PTFE) [7-9], poly(tetrafluoroethyleneco-hexafluropropylene) (FEP) [10-19], poly(ethylene-alt-tetrafluoroethylene) (ETFE) [20,21] and poly (vinylidene fluoride) (PVDF) [22-25] have been extensively used as substrates to develop sulfonic acid membranes by grafting of styrene or styrene/crosslinker mixtures onto them followed by sulfonation reactions.

In the example above, the writer used the citations to identify the studies which had investigated those specific materials and at the same time, to establish a link between studies with similar focus. Therefore, it serves two functions: identification and establishing links. 
The temperature at which the NOx conversion reached its maximum (673 K) corresponds to the temperature where nearly maximum oxidation of the C3H6 was achieved as observed in Cu/ZSM-5 [49,50], Cu/Al2O3 [2,5,51], and Ag/Y - Al2O3 [35].

In the second example, the citations are even used with three different functions: first to make a comparison between one's own findings and previous research (identified by the use of 'as'); second to establish a link between the studies with similar findings, and third to identify the studies which have investigated some specific material.

\subsection{CITATION TASKS}

After familiarizing students with different rhetorical functions of citations, they are given an exercise to identify the citation functions. This type of exercise is characterized in Task 1.

\subsection{Task 1}

Read the examples below and identify the rhetorical function(s) of citations in the column provided. You may assign more than one function to a citation. Some examples of citation functions are Attribution, Comparison, Support, Identification, etc.

Table 1 Task 1: Identifying Citation Functions

\begin{tabular}{|l|l|}
\hline \multicolumn{1}{|c|}{ Citations } & Rhetorical function(s) \\
\hline $\begin{array}{l}\text { 1. Muraki } \text { et al. [12] discovered that the platinum-modified CeO2 } \\
\text { catalysts were able to reduce NOx in a wide range of temperatures } \\
\text { under lean conditions. }\end{array}$ & \\
\hline $\begin{array}{l}\text { 2. Many studies have proven that cellulose acetate is highly } \\
\text { comparable to other synthetic polymer materials as well as effective } \\
\text { in hemodialysis process [23-27]. }\end{array}$ & \\
\hline $\begin{array}{l}\text { 3. These results were in agreement with those reported by Bahrami } \\
\text { et al. [4]. }\end{array}$ & \\
\hline $\begin{array}{l}\text { 4. Various kinds of metal oxide-supported Cu catalysts have been } \\
\text { thoroughly investigated in the SCR-HC such as Cu/SiO2 [3,4], } \\
\text { Cu/Al2O3 [5-7] and Cu/ZrO2 [8,9]. }\end{array}$ & \\
\hline $\begin{array}{l}\text { 5. Similar findings were reported by Kesting [18,19] on the effect of } \\
\text { inorganic salt additives upon the formation and properties of } \\
\text { cellulose acetate membranes. }\end{array}$ & \\
\hline $\begin{array}{l}\text { 6. Generally, the well-dispersed metal cocations will exhibit better } \\
\text { promoting results as reported by the studies over AgCl/Al2O3 [46], } \\
\text { Ag/Al2O3 [47], and Co/ZrO2 [48]. }\end{array}$ & \\
\hline
\end{tabular}


7. This trend observed could also be related to the viscosity of the solution and polymer hydrophilicity. It was reported that the presence of $\mathrm{LiBr}$ increased the viscosity of the solution [31].

8. The concentration of the feed and permeate solutions was determined by the method described elsewhere [33].

Answer key: 1 (Attribution), 2 (Establishing Links), 3 (Comparison), 4 (Identification/Establishing Links), 5 (Comparison/Establishing Links), 6 (Identification), 7 (Support/Identification), 8 (Reference).

Once the students are familiarized with different rhetorical functions of citations, they may be introduced to analysing the citations used in actual research articles in their own field of study. The students are asked to collect five articles from recognized journals in their field to serve as the basis for further textual analysis. In Task 2, while students are asked to identify different rhetorical functions of citations in the research papers, their attention is also drawn to the details of the citations, such as the number of total citations made, the age of citations, the most primary functions of citations, etc. If the students come from the same field of study, they may carry out this activity in groups; otherwise they perform the task individually.

\subsection{Task 2}

Select five published articles from different international journals in your own field of study. Identify all the citations in the papers. Answer the following questions. Remember that sometimes a citation may have more than one function.

1. How many citations are used in the whole paper? What is the average number of citations used for papers in the field?

2. How many citations are used in each section of the paper?

2. How many citations refer to studies within the current year of publication? How many citations referred to are less than 5 years old? More than 5 years old? 10 years old?

3. How many citations solely function as attribution?

4. How many citations are used to support the topic, methodology, findings and discussion?

6. How many citations are used to establish links between different sources? When are they used?

7. How many citations are used to provide reference for further information? When are they used? 
8. How many citations are used to make a comparison between one's own findings and that of the others? When are they used?

9. How many citations are used to identify the agent/ actor of the reporting verb or to identify studies which have investigated a particular material?

10. What are the prominent citation functions in the papers? What is the prominent citation function per section?

11. Can you identify any other citation functions in your sample articles?

Identify all the citation functions in your sample papers and write the number of each citation function in the chart below. The citations functions in Tables 2 and 3 are abbreviated as follows: Attri. refers to Attribution; Iden. refers to Identification; Sup. refers to Support; Ref. refers to Reference; Est. Link refers to Establishing Link; Comp. refers to Comparison; and Cit. refers to Citation.

Table 2 Task 2.1: Identifying Citation Functions in Selected Papers

\begin{tabular}{|l|l|l|l|l|l|l|l|l|l|}
\hline Samples & $\begin{array}{l}\text { No. of } \\
\text { Citation }\end{array}$ & Attri. & Iden. & Sup. & Ref. & Est. Link & Comp. & $\begin{array}{l}\text { Other } \\
\text { function }\end{array}$ & $\begin{array}{l}\text { No. of cit. } \\
\text { function }\end{array}$ \\
\hline Paper 1 & & & & & & & & & \\
\hline Paper 2 & & & & & & & & & \\
\hline Paper 3 & & & & & & & & & \\
\hline Paper 4 & & & & & & & & & \\
\hline Paper 5 & & & & & & & & & \\
\hline Total & & & & & & & & & \\
\hline
\end{tabular}

* since citations can have more than one function the total no. of citation functions are more than the total no. of citations

Now look at the citations in each section of the papers and complete the chart below. (The title of the sections in the papers might be different based on your field of study).

Table 3 Task 2.2: Identifying Citation Functions across Sections in Research Papers

\begin{tabular}{|l|l|l|l|l|l|l|l|l|l|}
\hline Sections & $\begin{array}{l}\text { No. of } \\
\text { Citation }\end{array}$ & Attri. & Iden. & Sup. & Ref. & Est. Link & Comp. & $\begin{array}{l}\text { Other } \\
\text { function }\end{array}$ & $\begin{array}{l}\text { No. of cit. } \\
\text { function }\end{array}$ \\
\hline Introduction & & & & & & & & & \\
\hline $\begin{array}{l}\text { Experiment/ } \\
\text { Methods }\end{array}$ & & & & & & & & & \\
\hline $\begin{array}{l}\text { Results \& } \\
\text { Discussion }\end{array}$ & & & & & & & & & \\
\hline $\begin{array}{l}\text { Summary/ } \\
\text { Conclusion }\end{array}$ & & & & & & & & & \\
\hline Total & & & & & & & & & \\
\hline
\end{tabular}




\subsection{Group Work Activity}

Compare your findings with a friend who is from a different field of study.

How are your answers different?

By answering the above questions, the students consciously become aware of citation practices in their own field of study. This knowledge will be able to guide them on how/when to employ citations in their own writing. Through making comparisons with others from different areas, they will also be consciously aware of different citation practices across different disciplines or fields of study.

\subsection{Guiding Students on Reviewing their own Citations}

In the previous segments, the students were made aware of different rhetorical functions of citations. They became familiarized with the details of incorporating citations in writing. Following Swales and Feak (2010), the final stage will have students reflect on their own writing practices; students are tasked to review their own citations and apply what they have learned in real situation. The students are given a checklist which will guide them to review the citations they have used in their own research paper. This checklist can be used by the students as a guide or it can be used in class as an additional activity.

\section{Use the checklist below to review your own citations}

$1 \quad$ Have I cited a sufficient number of sources in each section of my paper?

$2 \quad$ Have I cited both old and recent research in my area?

3 Have I used citations to support the topic of my study, my method segment, my findings and discussion?

$4 \quad$ Have I given prominence to support citations in the discussion section of my paper?

$5 \quad$ Have I compared my findings with previous research?

6 Have I used citations to show both similarities and dissimilarities with previous research?

$7 \quad$ Have I established links between different sources in my citations to show my knowledge of the field and to make my argument stronger?

8 Have I used Identification citations to report various studies with similar focus or findings?

$9 \quad$ Have I checked my citations with the source texts to see if I have cited them accurately?

10 Have I included all the cited sources in my reference list? 


\subsection{FINAL THOUGHT}

Using this model, we believe that beginning research writers can actually be trained to be text analysts for their own field through investigating and learning specific disciplinary textual practices for themselves, while the instructor acts mostly as the facilitator and guides them towards understanding different citation choices and strategies. The proposed materials were obviously designed with post-graduate students in mind, who these days more often than not, are forced to write for publication as part of their degree requirement. The tasks, however, could be modified based on the students' level. The model could also be applied for teaching other aspects or features of academic writing such as summarizing and paraphrasing, reporting verbs, and others.

Practice is of course a key factor in the success of writing. In EAP courses, instructors can give their students various writing assignments where they actually have the opportunity to incorporate multiple features of academic writing skills. For instance, the instructor may ask students to select a few related articles in their discipline and to write a short review paper citing information only from those selected articles using paraphrases, summaries and generalization as different ways of incorporating source texts in their writing. Students can be asked to submit both the review paper as well as the sources used which could easily be done these days with the help on the ubiquitous online learning system. Through this kind of writing practices, students would have to think strategically how to employ citations appropriately-they will be forced to incorporate information from the source texts in their writing, and at the same time, will try to apply the appropriate strategies since the source texts are available for comparison. They could even be asked to highlight the parts of the source texts that they have used in their citations. This "literature-based report" (Dovey, 2010, p. 54) is argued to be a type of writing task which can develop essential academic writing skills.

Advanced academic writing is complex and multifaceted. Faced with its complex features, uninitiated novice writers may easily become bewildered that some may resort to strategies which are not in line with appropriate or even accepted writing conventions. EAP instructors thus play an important role of raising students' awareness and highlighting the important writing features within and across fields of studies by first becoming informed themselves of various disciplinary writing conventions. Raising students' awareness of the textual or rhetorical features may perhaps make them more appreciative that advanced academic writing is a necessary competence that need to be consciously built and practiced over time. This paper has focused only on citation skill which is one of the important features in advanced academic writing. We believe that the materials and tasks offered here could be modelled after and similarly employed to teach closely related academic writing skills such as techniques of incorporating texts of others when paraphrasing, summarizing, and generalizing information; using various reporting verbs when reviewing the literature and so much more. 


\section{REFERENCES}

Abasi, A. R., Akbari, N. \& Graves, B. 2006. Discourse Appropriation, Construction of Identities, and the Complex Issue of Plagiarism: ESL Student Writing in Graduate School. Journal of Second Language Writing. 15(2): 102-117.

Abasi, A. R. \& Akbari, N. 2008. Are We Encouraging Patchwriting? Reconsidering the Role of Pedagogical Context in ESL Students' Transgressive Intertextuality. English for Specific Purposes. 27(30): 267-284.

Adler-Kassner, L., Anson, C. M. \& Howard, R. M. 2008. Framing Plagiarism. In Caroline Eisner and Martha Vicinus (Eds.). Originality, Imitation, and Plagiarism: Teaching Writing in the Digital Age (pp.231-246). Ann Arbor: University of Michigan Press.

Ahmad, U. K., Mansourizadeh, K., Koh, G. M. A. 2012. Non-native University Students' Perception of Plagiarism. Journal of Advances in Language and Literary Studies. 3(1): 39-48.

Bazerman, C. 1988. Shaping Written Knowledge. Madison: University of Wisconsin Press.

Bazerman, C., Little, J., Bethel, L., Chavkin, T., Fouquette, D., \& Garufis, J. 2005. Reference Guide to Writing across the Curriculum. West Lafayette, Indiana: Parlor Press.

Carroll, J. 2004. Institutional Issues in Deterring, Detecting and Dealing with Student Plagiarism. Joint Information Systems Committee. JISC Briefing Paper. Retrieved January 28, 2009 from: http://www.jisc.ac.uk/uploaded_documents/plagFinal.pdf.

Charles, M. 2006. Phraseological Patterns in Reporting Clauses Used in Citation: A Corpus-based Study of Theses in Two Disciplines. English for Specific Purposes. 25(3): 310-331.

Chau, M., H. 2017. More than Words: Text Matters in Language Learning. Malaysian Journal of Languages and Linguistics. 6(2): 1-16.

Dovey, T. 2010. Facilitating Writing from Sources: A Focus on Both Process and Product. Journal of English for Academic Purposes. 9(1): 45-60.

Flowerdew, J. 1999. Writing for Scholarly Publication in English: The Case of Hong Kong. Journal of Second Language Writing. 8(2): 123-145.

Flowerdew, J. \& Li, Y. 2007. Language Re-use among Chinese Apprentice Scientists Writing for Publication. Applied Linguistics. 28(3): 440-465.

Harwood, N. 2009. An Interview-based study of the Functions of Citation in Academic Writing Across Two Disciplines. Journal of Pragmatics. 41(3): 497-518.

Hewings, A. Lillis, T., Vladimirou, D. 2010. Who's Citing Whose Writings? A Corpus Based Study of Citations as Interpersonal Resource in English Medium National and English Medium International Journals. Journal of English for Academic Purposes. 9(2): 102-115.

Howard, R. M. 2002. Don't Police Plagiarism: Just TEACH! Education Digest. 67(5): 46-49.

Howard, R. M. 2007. Understanding "Internet Plagiarism". Computers and Composition. 24(1): 3-15.

Hyland, K. 1996. Writing Without Conviction? Hedging in Science Research Articles. Applied Linguistics. 17(4): 433-454.

Hyland, K. 1998. Persuasion and Context: The Pragmatics of Academic Metadiscourse. Journal of Pragmatics. 30(4): 437-455.

Hyland, K. 1999. Academic Attribution: Citation and the Construction of Disciplinary Knowledge. Applied Linguistics. 20(3): 341-367. 
Hyland, K. 2000. Disciplinary Discourses: Social Interactions in Academic Writing. Harlow: Longman. Johns, A. M. \& Swales, J. M. 2002. Literacy and Disciplinary Practices: Opening and Closing Perspectives. Journal of English for Academic Purposes. 1(1): 13-28.

Keck, C. 2006. The Use of Paraphrases in Summary Writing: A Comparison of L1 and L2 Writers. Journal of Second Language Writing. 15(4): 261-278.

Latour, B. \& Woolgar, S. 1979. Laboratory Life: The Social Construction of Scientific Facts. Beverly Hills: Sage.

Lave, J. \& Wenger, E. 1991. Situated Learning: Legitimate Peripheral Participation. Cambridge, UK: Cambridge University Press.

Li, Q. \& Zhang, X. 2021. An Analysis of Citations in Chinese English-major Master's Theses and Doctoral Dissertations. Journal of English for Academic Purposes. 51. (https://doi.org/10.1016/j.jeap.2021.100982)

Mansourizadeh, K. \& Ahmad, U. K. 2011. Citation Practices Among Expert and Novice Scientific Writers. Journal of English for Academic Purposes. 10(3): 152-161.

Mansourizadeh, K. \& Ahmad, U. K. 2016. An Investigation of Source Use Strategies in Published Research Articles and Graduate Students' Research Papers. Asian ESP Journal. 12 (1): 148182.

Myers, G. 1990. Writing Biology: Texts in the Social Construction of Scientific Knowledge. Madison: University of Wisconsin Press.

Pecorari, D. 2003. Good and Original: Plagiarism and Patchwriting in Academic Second-language Writing. Journal of Second Language Writing. 12(4): 317-345.

Pecorari, D. 2006. Visible and Occluded Citation Features in Postgraduate Second-language Writing. English for Specific Purposes. 25(1): 4-29.

Petrić, B. 2007. Rhetorical Functions of Citations in High- and Low-rated Master's Theses. Journal of English for Academic Purposes. 6(3): 238-253.

Robillard, A. E. \& Howard, R. M.2008. Introduction: Plagiarisms. In Rebecca Moore Howard and Amy E. Robillard (Eds.). Pluralizing Plagiarism: Identities, Contexts, Pedagogies (pp. 1-7). Portsmouth, NH: Heinemann Boynton/Cook.

Shi, L. 2010. Textual Appropriation and Citing Behaviours of University Undergraduates. Applied Linguistics. 31(1): 1-24.

Shooshtari, Z. G., Jalilifar, A., Shahri, S. 2017. Ethnolinguistic Influence on Citation in English and Persian Hard and Soft Science Research Articles. 3L: The Southeast Asian Journal of English Language Studies. 23(2): 58-74.

Swales, J. M. 1990. Genre Analysis: English in Academic and Research Setting. Cambridge: Cambridge University Press.

Swales, J. M. 2004. Research Genres: Explorations and Applications. Cambridge: Cambridge University Press.

Swales, J. \& C. Feak. 2010. "From Text to Task: Putting Research on Abstracts to work" in M. RuizGarrido, J. C. Palmer-Silveira \& I. Fortanet-Gómez (Eds.). English for Professional and Academic Purposes. Amsterdam: Rodopi. (pp. 167-180). 
Thompson, P. 2000. Citation Practices in PhD Theses. In L.Burnard \& T. McEnery (Eds.). Rethinking Language Pedagogy from a Corpus Perspective (pp. 91-101). Frankfurt: Peter Lang.

Thompson, P. 2005. Points of Focus And Position: Intertextual Reference in PhD Theses. Journal of English for Academic Purposes. 4(4): 307-323.

Thompson, P. \& Tribble, C. 2001. Looking at Citations: Using Corpora in English for Academic Purposes. Language Learning \& Technology. 5(3): 91-105.

Wenger, E. 1998. Communities of Practice: Learning, Meaning, and Identity. Cambridge University Press, Cambridge.

Zaid, Y. H., Shamsudin, S., Habil, H. 2017. Exploring Citations In Chemical Engineering Literature Review. LSP International Journal. 4(1): 55-67. 


\section{Appendix A}

List of the journals from which published research papers are sampled:

1. Separation and Purification Technology

2. Materials Science and Engineering

3. Applied Surface Science

4. Journal of Membrane Science

5. Journal of Catalysis 